\title{
A Special Topic on Nuclear Mechanobiology
}

This special topic is dedicated to the mechanics and structure of the cell nucleus, the importance of which has blossomed from the confluence of numerous fields. From the medical field, histological pathology utilized the nucleus as a diagnostic tool since it could be easily labeled and viewed, and nuclear size and morphology correlated with disease states. More recently, there has been interest in the mechanisms of disease progression related to these altered morphological states of the region of the cell where the genome is maintained. From the field of classical cell biology and genetics, there have been increasingly numerous human diseases associated with mutations in nuclear structural proteins including lamins and lamin-binding proteins. These diseases impact widely different tissue types, even when the mutations occur within the same protein, and the etiology has not been easily explained without considering the influences of structure and mechanics. From the technological fields of cellular and tissue engineering it was observed that there was an integrated chemical-mechanical feedback between cells and their engineered extracellular environment. In a desire to control and regulate cellular function, understanding the chemical and mechanical cues that dictate genome regulation and cell fate is critical. Consequently, the study of the mechanobiology of the cell nucleus has become an active and fruitful area of research marrying numerous fields in an attempt to understand both how forces and biochemical signaling are coordinated to modulate genome activity and homeostasis and how defects in these processes may impact human disease pathogenesis.

\section{NUCLEAR-CYTOSKELETAL COUPLING VIA THE LINC COMPLEX AND NUCLEAR PORE COMPLEXES}

The linker of nucleoskeleton and cytoskeleton (LINC) complex is considered to be the primary structural coupling across the nuclear envelope. ${ }^{1,14,17}$ First identified in 2005 by Crisp et al., the LINC complex consists of a series of proteins containing KASH-domain and SUN-domain proteins, which reside in the outer and inner nuclear membranes, respectively. ${ }^{7,18}$ This evolutionarily conserved molecular bridge is known to transmit spatial, structural and mechanical information across from the cytoskeleton to the nucleoskeleton. ${ }^{6,10,16}$ Numerous diseases have been shown to be associated with mutations in LINC complex proteins, typically in structural and mechanical tissues such as muscle. ${ }^{11,17,19}$ In "LINCing defective nuclear-cytoskeletal coupling and DYT1 dystonia", Saunders and Luxton consider how the pathogenesis of the neurological movement disorder DYT1 dystonia might result from dysfunctional regulation of the LINC complex. DYT1 dystonia is caused by a loss-of-function mutation in the ATPases associated with various cellular activities $(\mathrm{AAA}+$ ) protein, torsinA, which resides within the shared lumen of the endoplasmic reticulum and nuclear envelope. This review also emphasizes the importance of studying the complexities of the regulatory biology associated with living systems as well as the biophysics of force propagation through integrated cellular structures. Both this review and the one by Irianto et al. on cancer summarized below provide examples of a growing number of "nuclear mechanopathies", human diseases related to defects in nuclear mechanotransduction.

In "On the nuclear pore complex and its roles in nucleo-cytoskeletal coupling and mechanobiology" Soheilypour and colleagues conjecture that like the LINC complex, nuclear pore complexes (NPCs), which also bridge the cytoplasm and nucleoplasm, play a structural role in interconnecting the cytoskeleton with the nucleoskeleton. ${ }^{15,20}$ The authors investigate the known roles of different nuclear pore proteins, ${ }^{13}$ their location within the nuclear pore complex, and their known binding partners to propose a possible map of structural interactions across the NPC. They further provide evidence supporting the claim that the NPC acts as a complementary mechanical structure to the LINC complex and may also influence post-mitotic nuclear assembly. Since the analysis of many aspects of nuclear structure have been limited to interphase cells, it will be exciting to further pursue the role of these structural elements during other cell cycle-specific events such as nuclear envelope breakdown and reassembly during prometaphase and telophase, respectively.

In "O-GlcNAc-ylation in the Nuclear Pore Complex", Ruba and Yang examine the role of enzymatic modifications of nuclear pore proteins and how these alterations impact NPC structure and function. Serine and threonine residues present in numerous cytoplasmic and nuclear proteins can be modified by the 
addition of a specific carbohydrate, the O-linked glycan O-GlcNac, through a process known as "OGlcNA-cylation". ${ }^{3}$ Unlike classical protein glycosylation, O-GlcNAc-ylation is more similar to protein phosphorylation in that both modifications are dynamic and occur in a binary fashion. ${ }^{12}$ Together, Ruba and Yang critically examine recent literature, which has determined that over half of the thirty Nup proteins that make up the NPC are modified with this Olinked glycan. Of these proteins, most are considered intrinsically disordered and contain multiple phenylalanine-glycine $(\mathrm{FG})$ repeats. It is well known that FG repeat-containing Nups perform a barrier function that serves to control the selective diffusion of small $(<40 \mathrm{kDa})$ signal-independent cargo through the NPC. The authors propound that O-linked glycanmodified Nups may not perform a specific molecular function in the NPC per se. Rather, the presence of this O-linked glycan might alter the physical behavior of FG-repeat-containing Nups, which could increase their fluidity and therefore decrease their tendency to congeal due to hydrophobic forces. Ruba and Yang further offer an interesting "balance" model to explain the effect of O-linked glycan-modified Nups on NPC selective permeability. Since a major function of this post-translational modification is to prevent kinases from accessing and phosphorylating serine and threonine residues in proteins, ${ }^{3,12}$ it will be exciting in the future to consider the potential for regulatory modulation by crosstalk between Nup O-GlcNAc-ylation and phosphorylation. The relationship between glycobiology and cellular mechanics, which is highlighted briefly in this review, is destined to become a stimulating field of research in its own right.

\section{MORE THAN JUST A BARRIER: THE MEMBRANES OF THE NUCLEAR ENVELOPE}

Similar to many other cellular organelles, the nucleus is membrane-bound. However, the unique aspects of its structure - the contiguous double membrane, the large nuclear pore complexes, and the connection with the endoplasmic reticulum - strongly suggest the existence of distinctive functions and regulatory mechanisms not found in other organelles. ${ }^{21}$ In this topic the mechanochemical aspects that are unique to the nuclear membranes are discussed in detail. "Roles of cross-membrane transport and signaling in the maintenance of cellular homeostasis" by Cho and colleagues considers the similarities and differences in the mechanisms of transport of materials through the plasma membrane, the ER, and the nuclear membranes during cellular homeostasis. This review compares and contrasts the movement of ions and other chemical signals through membranes with the transduction of information via mechanical forces. The differences in speed and specificity between these chemical and mechanical signals indicate that the mechanotransduction of forces may enhance or complement chemical pathways that transmit information from the extracellular environment through the cytoplasm and into the nucleus.

In addition to the unique transport properties of the nuclear membranes, the mechanical functionality of these membranes had previously been poorly addressed. While the mechanosensitivity and tensionmediated regulation (via endocytosis and exocytosis as well as cytoskeletal regulation) of the plasma membrane is well known, the nuclear membranes have been largely ignored. In "A case for the nuclear membrane as a mechanotransducer", Enyedi and Niethammer present new evidence for their hypothesis that the nuclear membranes are mechanosensitive. They consider recently published evidence for the ability of the nuclear membranes to sense and transduce mechanical signals in an analogous fashion to the plasma membrane. This mechanosensitivity was indirectly identified by pathophysiology, and the mechanisms are just now being identified. In addition, the authors identify candidate mediators of nuclear membrane mechanosensitivity in an effort to spur on future mechanism-driven investigations.

The review by Torbati and colleagues entitled "An unresolved LINC in the nuclear envelope" provides a unique perspective on the spacing between the inner and outer nuclear membranes of the nuclear envelope. In this review, the authors critically evaluate decadesold experimental evidence for the maintenance of the 40-50 $\mathrm{nm}$ spacing between these membranes. They also argue the need for a balance of forces between the biophysical properties of membranes and the nuclear envelope-spanning structures of the LINC complex and NPCs. Based on thermodynamics theory and the presence of molecular motor-generated forces, a driving force must exist that is responsible for membrane fluctuation, curvature and fusion should exist, which would prevent this $40-50 \mathrm{~nm}$ spacing between the membranes of the nuclear envelope during interphase. Based on evidence from the literature, Torbati and colleagues propose the mechanical stability of LINC complexes and NPCs spread throughout the nuclear envelope balances out the forces produced by the mechanics of the nuclear membranes. Thus, LINC complexes and NPCs not only perform a nuclear-cytoskeletal coupling function, but also act together to stabilize the nuclear membranes. This mechanism of two-dimensional planar stabilization may prove to be particularly important for maintaining cellular function, especially when considered in the context of the 
reviews found in this special topic on nuclear membrane mechanosensitivity and transport. The ideas presented in this review are intriguing when considered in light of recently published work from the Starr laboratory, which demonstrates that in Caenorhabditis elegans LINC complexes are only required for the maintenance of nuclear membrane spacing in cells exposed to increased mechanical forces, such as muscle cells. ${ }^{5}$ It will be exciting to watch the resolution of these important questions over the next few years.

\section{WHAT LIES BENEATH?}

The nucleoskeleton, which underlies the inner nuclear membrane, is largely comprised of nuclear lamins and their associated proteins. ${ }^{4}$ In "Nuclear lamins in cancer", Irianto and colleagues review the increasingly accepted role of the nucleoskeletal changes that are associated with several cancers. Alterations in nuclear size and morphology as well as abnormal chromatin organization and distribution are the basic microscopic criteria used by pathologists for the diagnosis of numerous cancers. ${ }^{8,9}$ Regarding the correlation between nucleoskeleton alterations and cancer, cancerous cells exhibit changes in the levels of lamin expression and/or stability, the ratio of A-type versus B-type lamins, alterations in the amount and type of lamin posttranslational modifications, as well as various similar changes in lamin-associated proteins. These modifications are associated with altered chemical signaling pathways, genomic structural dysregulation, and altered cellular mechanics. Interestingly, such alterations in nuclear mechanics may contribute to the metastatic and invasive potential of cancer cells. Finally, the authors draw attention to how the load bearing structures of the cell work in homeostatic balance with the extracellular matrix to sense matrix rigidity, which may also be a confounding factor during carcinogenesis.

Within the nucleus is the genome, which is a hyperorganized DNA structure including all of the necessary enzymatic and regulatory components for phenotype control. $^{2}$ In "Mechanobiology of chromatin and the nuclear interior", Spagnol and colleagues introduce the different levels of self-assembled organization inside the nucleus including DNA structures and nuclear bodies. Specifically, the authors discuss how these elements are functionally reorganized by the mechanotransduction of forces generated within the cytoplasm by the cytoskeleton. Spagnol et al. also examine the effect of chemical stimulation on nuclear structure and function as established throughout recent literature. In addition, this review underscores the fascinating intersection between nuclear mechanics and regulated gene expression. The authors advise that a combination of cellular biology, mechanics, and polymer physics is necessary to begin to unravel the mechanisms underlying the mechanotransduction of chemical and physical stimuli across the nuclear envelope and into the nucleoplasm will significantly advance our understanding of the mechanical modulation of gene expression.

\section{CONCLUSIONS, COMMON THEMES AND MOVING FORWARD}

While the concept of the nucleus is no longer that of a lone, isolated organelle floating freely within the cytoplasm, it is clear that we have only just begun to appreciate the mechanobiology of the nucleus and its dynamic integration with the remainder of the mechanical cell. Recurrent themes throughout this special topic include complex mechanical and chemical regulation of nuclear structures: the chromatin, nuclear membranes, nuclear envelope-spanning protein complexes, and nucleoskeleton. Another common theme is the balance of seemingly opposing entropic driving forces imposed by physical fluctuations of biomacromolecules and self-assembly with the order imposed by the biology of the system. These antagonistic aspects lead to the evolution of a pseudostable structure that allows for the proper functioning of this important organelle while allowing for sufficient levels of instability, which is critical for the ability of the nucleus to respond and adapt when necessary. Taken together the extent of these daunting levels of complexity demand that researchers apply an interdisciplinary approach in order to even attempt to fully understand the amazing machinery of the cell.

Be sure to follow the latest developments in Cellular and Molecular Bioengineering on Facebook (http:// www.facebook.com/CMBEjournal) and Twitter (http:// www.twitter.com/CMBEjournal).

\section{ACKNOWLEDGMENTS}

The authors would like to thank Donald R. Mattison, McLaughlin Center for Population Health Risk Assessment, University of Ottawa, Ottawa, ON, Canada, for his comments and review of an earlier version of this article.

\section{REFERENCES}

${ }^{1}$ Alam, S., D. B. Lovett, R. B. Dickinson, K. J. Roux, and T. P. Lele. Nuclear forces and cell mechanosensing. Prog. Mol. Biol. Transl. Sci. 126:205-215, 2014.

${ }^{2}$ Babu, D., and M. J. Fullwood. 3D genome organization in health and disease: emerging opportunities in cancer translational medicine. Nucleus 6:382-393, 2015. 
${ }^{3}$ Bond, M. R., and J. A. Hanover. A little sugar goes a long way: the cell biology of O-GlcNAc. J. Cell Biol. 208:869880, 2015.

${ }^{4}$ Burke, B., and C. L. Stewart. The nuclear lamins: flexibility in function. Nat. Rev. Mol. Cell Biol. 14:13-24, 2013.

${ }^{5}$ Cain, N. E., E. C. Tapley, K. L. McDonald, B. M. Cain, and D. A. Starr. The SUN protein UNC-84 is required only in force-bearing cells to maintain nuclear envelope architecture. J. Cell Biol. 206:163-172, 2014.

${ }^{6}$ Chambliss, A. B., et al. The LINC-anchored actin cap connects the extracellular milieu to the nucleus for ultrafast mechanotransduction. Sci. Rep. 3:1087, 2013.

${ }^{7}$ Crisp, M., et al. Coupling of the nucleus and cytoplasm: role of the LINC complex. J. Cell Biol. 172:41-53, 2006.

${ }^{8}$ de Las Heras, J. I., D. G. Batrakou, and E. C. Schirmer. Cancer biology and the nuclear envelope: a convoluted relationship. Semin. Cancer Biol. 23:125-137, 2013.

${ }^{9}$ Fischer, A. H. The diagnostic pathology of the nuclear envelope in human cancers. Adv. Exp. Med. Biol. 773:49 75,2014

${ }^{10} \mathrm{Guilluy}, \mathrm{C}$., et al. Isolated nuclei adapt to force and reveal a mechanotransduction pathway in the nucleus. Nat. Cell Biol. 16:376-381, 2014.

${ }^{11}$ Horn, H. F. LINC complex proteins in development and disease. Curr. Top. Dev. Biol. 109:287-321, 2014.

${ }^{12} \mathrm{Hu}, \mathrm{P}$., S. Shimoji, and G. W. Hart. Site-specific interplay between O-GlcNAcylation and phosphorylation in cellular regulation. FEBS Lett. 584:2526-2538, 2010.

${ }^{13}$ Ibarra, A., and M. W. Hetzer. Nuclear pore proteins and the control of genome functions. Genes Dev. 29:337-349, 2015.

${ }^{14}$ Jahed, Z., H. Shams, M. Mehrbod, and M. R. Mofrad. Mechanotransduction pathways linking the extracellular matrix to the nucleus. Int. Rev. Cell Mol. Biol. 310:171-220, 2014.

${ }^{15}$ Knockenhauer, K. E., and T. U. Schwartz. The nuclear pore complex as a flexible and dynamic gate. Cell 164:1162-1171, 2016.
${ }^{16}$ Lombardi, M. L., et al. The interaction between nesprins and sun proteins at the nuclear envelope is critical for force transmission between the nucleus and cytoskeleton. J. Biol. Chem. 286:26743-26753, 2011.

${ }^{17}$ Luxton, G. W., and D. A. Starr. KASHing up with the nucleus: novel functional roles of KASH proteins at the cytoplasmic surface of the nucleus. Curr. Opin. Cell Biol. 28:69-75, 2014.

${ }^{18}$ Meinke, P., and E. C. Schirmer. LINC'ing form and function at the nuclear envelope. FEBS Lett. 589:25142521, 2015.

${ }^{19}$ Meinke, P., T. D. Nguyen, and M. S. Wehnert. The LINC complex and human disease. Biochem. Soc. Trans. 39:16931697, 2011.

${ }^{20}$ Simon, D. N., and K. L. Wilson. The nucleoskeleton as a genome-associated dynamic 'network of networks'. Nat. Rev. Mol. Cell Biol. 12:695-708, 2011.

${ }^{21}$ Wilson, K. L., and S. C. Dawson. Evolution: functional evolution of nuclear structure. J. Cell Biol. 195:171-181, 2011.

\section{Kris Noel DAHL}

Department of Biomedical Engineering, Department of Chemical Engineering Carnegie Mellon University, Pittsburgh, PA, USA

Electronic mail: krisdahl@cmu.edu

\section{G. W. Gant LuXton}

Department of Genetics, Cell Biology, and Development, University of Minnesota, Minneapolis, MN, USA 